\title{
Incorporating real time velocity map image reconstruction into closed-loop coherent control
}

\author{
C. E. Rallis, ${ }^{1}$ T. G. Burwitz, ${ }^{1}$ P. R. Andrews, ${ }^{1}$ M. Zohrabi, ${ }^{2}$ R. Averin, ${ }^{1}$ S. De,${ }^{2, a)}$ \\ B. Bergues, ${ }^{3}$ Bethany Jochim, ${ }^{1,2}$ A. V. Voznyuk, ${ }^{1}$ Neal Gregerson, ${ }^{1}$ B. Gaire, ${ }^{2, b)}$ \\ I. Znakovskaya, ${ }^{3}$ J. McKenna, ${ }^{2}$ K. D. Carnes, ${ }^{2}$ M. F. Kling, ${ }^{2,3}$ I. Ben-Itzhak, ${ }^{2}$ and E. Wells ${ }^{1, c}$ \\ ${ }^{1}$ Department of Physics, Augustana College, Sioux Falls, South Dakota 57197, USA \\ ${ }^{2}$ J.R. Macdonald Laboratory, Department of Physics, Kansas State University, Manhattan, Kansas 66506, USA \\ ${ }^{3}$ Max Planck Institute of Quantum Optics, Hans-Kopfermann Strasse 1, D-85748 Garching, Germany
}

(Received 15 July 2014; accepted 13 October 2014; published online 7 November 2014)

\begin{abstract}
We report techniques developed to utilize three-dimensional momentum information as feedback in adaptive femtosecond control of molecular dynamics. Velocity map imaging is used to obtain the three-dimensional momentum map of the dissociating ions following interaction with a shaped intense ultrafast laser pulse. In order to recover robust feedback information, however, the twodimensional momentum projection from the detector must be inverted to reconstruct the full threedimensional momentum of the photofragments. These methods are typically slow or require manual inputs and are therefore accomplished offline after the images have been obtained. Using an algorithm based upon an "onion-peeling" (also known as "back projection") method, we are able to invert 1040 $\times 1054$ pixel images in under $1 \mathrm{~s}$. This rapid inversion allows the full photofragment momentum to be used as feedback in a closed-loop adaptive control scheme, in which a genetic algorithm tailors an ultrafast laser pulse to optimize a specific outcome. Examples of three-dimensional velocity map image based control applied to strong-field dissociation of $\mathrm{CO}$ and $\mathrm{O}_{2}$ are presented. (C) 2014 AIP Publishing LLC. [http://dx.doi.org/10.1063/1.4899267]
\end{abstract}

\section{INTRODUCTION}

The technique of adaptive femtosecond control, in which experimental feedback is used to guide an algorithm in the identification of a shaped ultrafast laser pulse that optimizes a particular atomic or molecular outcome, has been demonstrated in many experiments. ${ }^{1,2}$ In these measurementdriven schemes, intense ultrafast lasers equipped with pulseshaping devices ${ }^{3}$ produce pulses that control the dynamics of atoms and molecules. The optimal pulse is selected using an adaptive learning algorithm that depends on experimental feedback. Since the algorithm must search through a large number of possible pulses to arrive at an optimal solution, the feedback must be rapid. Typical experiments have relied on optical signatures such as fluorescence and Raman scattering, ${ }^{4,5}$ or monitored particle yield using time-of-flight mass spectrometry. ${ }^{6}$ These techniques tend to monitor the yield of a single variable as feedback.

Independent of adaptive femtosecond control, studies of the ionization and dissociation of molecules by photons and charged particles have been greatly advanced by technological developments that allow the measurement of correlated multi-dimensional data. A straightforward variation is a coincident time-of-flight technique, in which all the charged fragments from a dissociation are measured in event-mode, often uniquely identifying a particular dissociation channel. ${ }^{7-10}$ In the most sophisticated forms, multi-hit position sensitive de-

\footnotetext{
a) Present address: Saha Institute of Nuclear Physics, 1/AF Bidhannagar, Kolkata 700064, India.

b) Present address: Chemical Sciences Division, Lawrence Berkeley National Laboratory, Berkeley, California 94720, USA.

c) Electronic mail: eric.wells@augie.edu.
}

tectors are coupled with a cold target to produce kinematically complete measurements. ${ }^{11,12}$ Nearly all of these correlated multi-particle techniques produce event-mode data that are accumulated over many interactions since they are limited to one laser-molecule interaction/laser pulse. While there has been limited use of coincidence time-of-flight techniques to provide feedback for adaptive femtosecond control, ${ }^{13}$ in general many of these methods are too slow for efficient use in a feedback control loop. This is unfortunate, since ion-based feedback, in particular, cannot always target a well-defined objective for the learning algorithm to optimize. ${ }^{14}$

Velocity map imaging (VMI) ${ }^{15-17}$ represents an experimental compromise between the requirement for acquisition speed and the desire to target more specific final states. In this method, a spectrometer with a suitable electrostatic lens is used to focus all fragments with identical velocity components to a specific point on the detector, despite the fact that these ions may have originated from different locations. The expanding Newton sphere strikes a microchannel plate (MCP) detector producing electrons that are accelerated to a phosphor screen which is imaged with a charge-coupled device (CCD) camera. While individual events cannot be easily correlated with this method, VMI obtains fragments over $4 \pi$ steradians and the acquisition speed is usually limited by the detector. Thus, VMI provides angle-resolved kinetic energy release data for a given ion species at a rapid enough pace to be a candidate for use as closed-loop feedback. In fact, imaging studies of pulse-shape-dependent strong-field processes have appeared, ${ }^{18-21}$ and some initial image-based feedback techniques have already been used by various groups. ${ }^{21-24} \mathrm{In}$ these initial efforts, the raw two-dimensional (2D) image was used as feedback. 
Complete momentum information, however, cannot be obtained directly from the raw 2D VMI data itself, because of the reduction in dimensionality inherent in the projection of the three-dimensional (3D) Newton sphere onto the 2D detector plane. As a result, it is often difficult to understand the raw images well enough to identify useful regions for feedback. The main difficulty is the raw 2D image contains the superfluous azimuthal contribution. Furthermore, as the radius of the Newton sphere increases, the signal is spread over more area, making it difficult to visually compare the signal strength at small and large radii. These problems are not simply aesthetic. As discussed in Sec. VI, several of our early attempts to use 2D images as feedback failed because the azimuthal contribution from higher momentum photofragments masked the physical process with lower dissociation energy that could be used to reach the control objective. ${ }^{21}$

When the image has cylindrical symmetry, which in many cases is provided by the laser polarization axis, a number of methods ${ }^{17}$ are available to convert the raw $2 \mathrm{D}$ image into a slice of the full 3D momentum map. We have recently applied this ability to adaptive strong-field control of acetylene and ethylene. ${ }^{25}$ In the present article we describe our technique for incorporating full 3D momentum information into adaptive femtosecond control, including the details of our rapid inversion, our imaging system and data acquisition, and the computer control that manages the adaptive search. In our implementation, the image analysis time, including the inversion of the $2 \mathrm{D}$ image to recover a slice through the full $3 \mathrm{D}$ momentum distribution, has been reduced in duration $(<1 \mathrm{~s})$ to the point that it is less than the typical image acquisition time of $1-3 \mathrm{~s}$.

The ability to target more specific final states for optimization should be a powerful tool for reaching a better understanding of the mechanisms that undergird adaptive femtosecond control. These mechanistic questions are important because there is, so far, no general prescription for choosing relevant search parameters around which to structure the pulse characteristics. Poor choices of pulse parameters can lead to artificial traps in phase space, limiting the ability of the algorithm to find an optimal solution. ${ }^{26}$ The scheme presented below offers several advantages for experimenters seeking to combine learning algorithms with more specific ion-based feedback.

\section{EXPERIMENTAL APPARATUS}

Our experimental setup consists of an ultrafast laser system with a pulse shaper, a VMI spectrometer, and computer control that links them together and contains the genetic algorithm (GA) that uses the feedback signals to determine the optimal pulse shapes. A schematic of this configuration is shown in Fig. 1. The laser pulses are provided by a Ti:sapphire laser system with a multi-pass amplifier. The amplified pulses have a center wavelength of approximately $785 \mathrm{~nm}$, a pulse energy of up to $2 \mathrm{~mJ}$, a repetition rate of up to $2 \mathrm{kHz}$ and a Fourier transform-limited (FTL) duration of approximately $45 \mathrm{fs}$ with the pulse shaper placed between the oscillator and the amplifier. Typical pulse energies in these experiments were between 0.01 and $0.5 \mathrm{~mJ} /$ pulse, which corresponds to a peak intensity

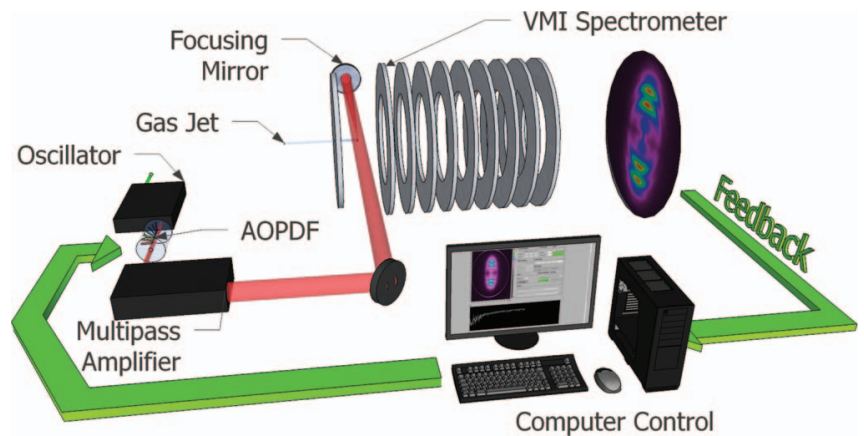

FIG. 1. Schematic of the experimental setup, illustrating the control loop. Shaped pulses from the AOPDF are amplified and focused into the gas jet inside the spectrometer. The ions are imaged using the VMI technique and that data is used to calculate a fitness function, which the learning algorithm uses to produce subsequent pulse shapes.

of up to $2 \times 10^{16} \mathrm{~W} / \mathrm{cm}^{2}$ after focusing by a $f=75 \mathrm{~mm}$ spherical mirror. The laser is linearly polarized and the polarization axis is vertical in Fig. 1 and along the $z$-axis as defined in Fig. 2. The polarization axis provides the cylindrical symmetry that allows the image inversion discussed in Sec. III. The laser pulse shaper is an acousto-optic programmable dispersive filter (AOPDF) ${ }^{27}$ that may be operated in several modes: shaping only the spectral phase, controlling the spectral amplitude, or combined phase-amplitude shaping. Most of our experiments are done with phase-only shaping. In this configuration, the pulse energies remain essentially constant over the duration of the adaptive control experiments, although the intensity is dependent on the pulse duration. Pulse shapes are monitored with a Second-Harmonic-Generation FrequencyResolved Optical Gating (SHG FROG) ${ }^{28}$ scheme.

The thick-lens VMI spectrometer used in these experiments has been described in other publications ${ }^{29-31}$ and contains an effusive jet to deliver the target gas, similar to the one described by Ghafur and co-workers. ${ }^{32}$ As shown in Fig. 1, the laser passes through the spectrometer and is focused by the mirror into the effusive gas target. Following ionization by the laser pulse, the ions are projected onto the microchannel plate-phosphor screen assembly and imaged by a

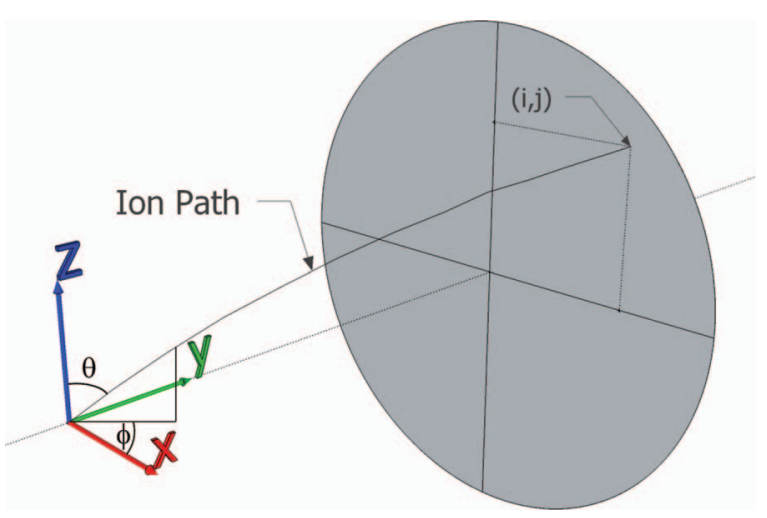

FIG. 2. Coordinate system used to describe the image inversion. The electric field of the spectrometer is in the $y$-direction, laser polarization is in the $z$ direction, and the laser propagates in the $x$-direction. The angle $\theta$ is the angle between the laser polarization direction $(z)$ and the velocity vector of the ion. The linear polarization dictates that the data is symmetric with rotation in $\phi$ about the $z$-axis. 
CCD camera. As is standard with the VMI technique, ${ }^{16,17}$ the spectrometer ion optics ensure that all ions emerging from the laser focus with the same velocity vector strike the detector in the same location. The base pressure in the VMI spectrometer chamber is below $1 \times 10^{-9}$ Torr. A precision leak valve feeds the effusive jet allowing the target density to be controlled above this baseline pressure.

The MCPs are fully powered only at times corresponding to the arrival of a single preselected ion species using a triggered fast high-voltage switch controlled by a digital delay generator linked to a photodiode that monitors the laser output for synchronization with the laser pulse. If desired, images of multiple ion species may be obtained by changing the trigger delay via the interface between the delay generator and the control computer.

A filter is placed between the phosphor screen and the camera to block stray reflections from the laser light and the entire detector and camera assembly is covered to reduce any background from ambient light. The phosphor screen is imaged with a cooled low-noise CCD camera (PCO sensicam qe) with $1040 \times 1054$ pixels and 12-bit dynamic range. In practice, the dynamic range of the camera usually determines the exposure time. This ion image is processed online (as described in Sec. III) and a particular "fitness" value is extracted, which is used by the learning algorithm (GA) to produce a new set of pulse shapes for testing. Details of the computer control linking the various aspects of the experiment are described in the Appendix.

\section{IMAGE RECONSTRUCTION}

The essential problem to be solved is illustrated in Fig. 3, which shows the VMI of $\mathrm{CD}_{3}^{+}$ions produced in laser-ethylene interactions. While the outer structure is visible in the raw image on the left, the interesting inner structure is nearly com-

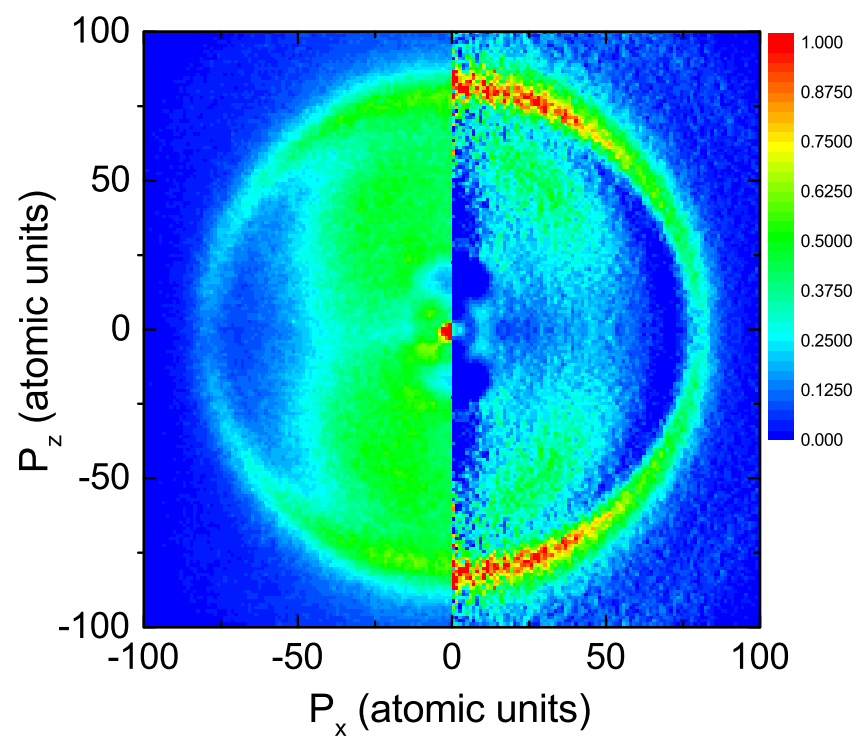

FIG. 3. (left) Raw 2D image of $\mathrm{CD}_{3}^{+}$ions following photofragmentation of $\mathrm{C}_{2} \mathrm{D}_{4}$ molecules by an intense laser pulse. (right) The resulting slice through the full 3D momentum distribution of the same data following symmetrization and inversion. The laser polarization is vertical in this image. pletely obscured. A robust feedback system should rapidly convert the raw 2D image on the left into the slice through the $3 \mathrm{D}$ distribution shown on the right, from which a well-defined gated yield may be derived. The desired yield is differential in momentum $\left(P_{z}\right.$ and $\left.P_{x}\right)$ or, equivalently, angle and kinetic energy release (KER) of the photofragments.

Mathematically, given the cylindrical symmetry conveniently provided by the laser polarization axis (aligned parallel to the VMI axis), accomplishing the image inversion requires performing an inverse Abel transformation. ${ }^{17,33-35} \mathrm{Ex}-$ plicitly, the 3D momentum (or velocity) distribution signal $p(x, y, z)$ is related to the measured ion distribution $q(x, z)$, by

$$
q(x, z)=\int_{-\infty}^{\infty} p(x, y, z) d y
$$

where the coordinate system is described in Fig. 2. Ultimately, we wish to recover a slice through the $3 \mathrm{D}$ momentum distribution with the azimuthal contribution removed. Starting with a single row of the image along the $x$-axis and perpendicular to the symmetry axis at some $z=z_{j}$, such that $s(x, y)$ $=p\left(x, y ; z_{j}\right)$,

$$
f(x)=q\left(x, z_{j}\right)=\int_{-\infty}^{\infty} s(x, y) d y=2 \int_{0}^{\infty} s(x, y) d y .
$$

Therefore, we must recover $s(x, y)$ from the measured distribution $f(x)$, where $f(x)$ is the function representing the slice through the image at $z_{j}$. Using the cylindrical symmetry of $s(x, y)$ provided by the laser polarization and switching to polar coordinates via the standard change of variables $r^{2}=x^{2}$ $+y^{2}$, we find that

$$
f(x)=2 \int_{x}^{\infty} \frac{s(r) r}{\sqrt{r^{2}-x^{2}}} d r .
$$

This is the Abel transform. ${ }^{33,35}$ The inverse transform is obtained by applying the Fourier transform convolution theorem. ${ }^{35}$ The quantity of interest is

$$
s(r)=-\frac{1}{\pi} \int_{r}^{\infty} \frac{d f / d x}{\sqrt{x^{2}-r^{2}}} d x,
$$

which gives a method of recovering the original distribution since we can obtain $s\left(r, z_{j}\right)$ for each row $f\left(x, z_{j}\right)$.

The solution of Eq. (4) is complicated by the singularity at $x^{2}=r^{2}$ and the fact that the $d f / d x$ term in the integral can magnify noise. ${ }^{17}$ A number of numerical methods of solving this problem exist, but not all of them are useful for the present inversion task due to the additional constraints of the adaptive control loop. First, since a typical experiment contains a few thousand trial pulses, the inversion cannot take much longer than the image acquisition time. Ideally, the inversion time will be much shorter. Second, since the image features are not known ahead of time, methods that require manual selection of initial parameters (such as an appropriate basis set) are difficult to implement. Finally, the inversion process should avoid introducing noise that compromises the feedback. 
TABLE I. Comparison of the different inversion methods investigated in this work. The tests were conducted with a $661 \times 661$ pixel test image that had been symmetrized before the inversion. The POP and Onion-Peeling tests used a $3.33 \mathrm{GHz}$ CPU on a Windows-based computer; the pBASEX and Iterative tests used a $2.4 \mathrm{GHz} \mathrm{CPU}$ on a Linux-based computer. The duration of the iterative routine depends on the number of cycles through the iterative algorithm. Here we selected a number of iterations $(\sim 35)$ that seemed to provide an image that was visually comparable to the other inversion methods and for which the reduction of the error (see Eq. (8) of the article by Vrakking ${ }^{45}$ ) between iterations began to diminish.

\begin{tabular}{lccl}
\hline \hline Method & $\begin{array}{c}\text { Calculation } \\
\text { time (s) }\end{array}$ & $\begin{array}{c}\text { Initial } \\
\text { parameters? }\end{array}$ & Noise \\
\hline $\begin{array}{l}\text { Fourier-Hankel }^{40} \\
\text { pBASEX }^{47}\end{array}$ & N/A & No & Yes \\
& $1.5 \pm 0.1$ & Yes & $\begin{array}{l}\text { Depends on } \\
\text { parameters } \\
\text { Iterative }\end{array}$ \\
POP $^{49}$ & $174 \pm 5$ & Somewhat & $\begin{array}{l}\text { Center spot } \\
\text { Negative } \\
\text { Onion-Peeling }\end{array}$ \\
\hline \hline
\end{tabular}

With these constraints in mind, it is worth reviewing existing options. First, the problems discussed above may be circumvented altogether by slice imaging techniques, ${ }^{36-38}$ in which the detector is only powered for a short time, thereby "slicing" a portion of the total momentum distribution. The resolution of this method is limited by the switching speed of the power supply and the detector size. The latter is important because if the extraction voltage is lowered to increase resolution in the time-of-flight direction, eventually ions will begin to miss the detector. This problem becomes more acute for lighter ions.

Within the realm of computational techniques, there are a large number of options. ${ }^{39-49}$ Each of these methods has attributes that make them attractive in various situations, but not all of them were suitable for our purposes. Table I summarizes the results of our testing. Fourier-Hankel based methods ${ }^{39-41}$ are fast but often result in increased noise near the center of the image where the Bessel function oscillates rapidly. ${ }^{17} \mathrm{Sev}-$ eral methods, such as BASEX ${ }^{46}$ and pBASEX, ${ }^{47}$ use functions that have known inverse Abel transformations. This relocates the centerline noise common to the Abel methods to the center point of the image and computes smooth images fairly quickly. The main drawback for our application is that they require the selection of a basis set prior to the experiment. The iterative method ${ }^{45}$ limits some of these problems but is too slow for our application. Hybrid methods, such as polar onion peeling (POP) ${ }^{49}$ and combining BASEX-like features with the iterative approach ${ }^{48}$ still require initial parameters to be determined. The POP algorithm occasionally yielded negative values in our tests, which complicates the determination of a yield-based fitness value.

For these reasons, we chose the "onion-peeling" (sometimes called "back-projection") method. ${ }^{17,42-44}$ While this technique does result in some centerline noise, the noise can be reduced to levels that do not interfere with the adaptive control by managing two experimental considerations. First, the image had to be sized on the detector so that the outer region was free, or nearly free, of signal. Due to the nature of the inversion, spurious signal in the outer region, from dark counts or stray reflections, leads to excessive subtractions in the center portion of the image. By reducing ambient light and acquiring a background image with no laser present and subtracting this from each experimental image during the run, this issue can be minimized. Second, care is needed in defining the center of the image. To assist with this, we plot raw images with a 1:1 ratio between camera pixels and screen display pixels and use these plots to determine the image center. Importantly, there are also no initial parameters to be specified, other than the center and size of the image. Our implementation of the onion-peeling procedure is derived from the algorithm described by Bordas and co-workers, ${ }^{42}$ and the interested reader is directed to their excellent development of the mathematical formalism.

Conceptually, the inversion works, as the onion-peeling name implies, by starting on the outside of the image and subtracting the contribution of ions with a particular kinetic energy from the entire image inside that point. Ions of mass $m$ that originate from the origin $(r=0)$ at time $t=0$ with a specific kinetic energy, $W$, are found at a later time $t$ to occupy a sphere of radius $r_{t}$, described by ${ }^{42}$

$$
W=\frac{m r_{t}^{2}}{2 t^{2}}=\gamma r_{t}^{2},
$$

where $\gamma=m / 2 t^{2}$. This means that the inversion must proceed from outside to inside because all ions with kinetic energy exceeding some threshold value $W_{1}=\gamma r_{1}^{2}$ can contribute to the image at radius $r_{1}$. By starting at the outside edge of the image at pixel $\left(i_{0}=i_{\max }, j_{0}\right)$, where $i_{\text {max }}$ is the largest pixel value, we assume that any signal on that pixel is attributed to ions emitted at $\phi=0$, or the maximum kinetic energy value $W_{1}=\gamma r_{1}^{2}$ associated with that radius. The signal at a given pixel $\left(i_{0}, j_{0}\right)$ allows the calculation of the total number of ions produced at angle $\phi$ with energy $W_{1}$. Thus, the number of ions with energy contributing to signal at pixels $i=1 \ldots i_{0}$ can be determined using the projection operator developed by Bordas et $a l .{ }^{42}$ The contribution is added to the back-projected slice that is being created [at pixel location $\left(i_{0}, j_{0}\right)$ ] and subtracted from the measured projection $q\left(i, j_{0}\right)$ for all $i \leq i_{0}$. The procedure is repeated for the next lower value until the center of the image is reached. Each line (i.e., $j$ value) of the image is treated separately, a fact that is made possible by the experimental condition that the electrostatic energy gained by the ions in the spectrometer is much larger than the inherent energy of the ions due to the laser-molecule interaction. ${ }^{42}$ In our case, the spectrometer voltages were at least $500 \mathrm{~V}$ and typically $1 \mathrm{kV}$ or more.

Computationally, we found that carrying out this procedure using a matrix-based MATLAB ${ }^{50}$ environment had several advantages. Perhaps most importantly, since the control code for the experiment was built in LabVIEW, ${ }^{51}$ calling a MATLAB "script node" was simple and variables and matrixformatted data could be quickly passed between the programs. In addition, expressing the image as a matrix allowed the use of some predefined functions, in some cases eliminating a for-loop. Careful optimization of the code reduced the computation time for our $661 \times 661$ pixel test image to $50 \mathrm{~ms}$, more than an order of magnitude faster than the $\mathrm{POP}^{49}$ or 
pBASEX ${ }^{47}$ methods we tested. For a full-sized $1040 \times 1054$ pixel image, the inversion time was still only around $0.6 \mathrm{~s}$, and the total image processing time (which includes background subtraction and possibly symmetrization) remained below 1 $\mathrm{s}$, below the image acquisition time (typically 1-2 s). Additional efficiency was gained by separating the image acquisition and processing steps by running them on separate cores of the CPU. Thus, while one image was being acquired, the previous image was being inverted, and as long as the total processing time remained less than the acquisition time, the length of the experiment was unaffected by the inversion.

Since each line of the image is inverted separately, there would seem to be some advantage in developing a parallelprocessing version of the code, perhaps even to the extent of running that section of the algorithm on a dedicated graphics processing unit (GPU). Preliminary investigations along these lines did not yield much improvement over the standard single thread application. The most likely reasons for this finding are the extra overhead related to parallel processing implementations and issues related to communication bandwidth and latency between the GPU and the CPU. Higher resolution cameras, however, may change the relative balance of these factors in the future.

The inversion of VMI data continues to be an active research subject. ${ }^{52-54}$ After the completion of the testing summarized in Table I, a statistical method based upon a maximum entropy concept was published. ${ }^{54}$ This technique (called MEVIR, for Maximum Entropy Velocity Image Reconstruction) limits noise and appears to produce robust images even with limited signal, making it an attractive candidate for further study. At the present time this method requires a reported $20-40$ iterations at $142 \mathrm{~ms} /$ iteration for a $600 \times 600$ pixel test image ${ }^{54}$ To be a viable alternative to the onion-peeling method in our experiments, the time to obtain the 3D-VMI would need to be reduced to around 1-2 s for the larger 1040 $\times 1054$ pixel images captured from our camera. As discussed in the Appendix, the modular nature of our computer control allows the inversion routine to be upgraded as the speed of the MEVIR approach is increased or other methods become available.

\section{DEFINING THE IMAGE-BASED FITNESS FUNCTION}

Once the image is inverted, regions can be defined on the slice through the 3D-VMI and the yield from these regions is used to calculate the fitness function (or control objective), which produces a value that the adaptive control optimizes. In our case, the fitness function can be defined on the graphical user interface of the control program. The fitness may depend on up to four regions of a single 3D-VMI, or up to two regions on 3D-VMI measurements of two different ion species. In principle, more species could be added to the fitness calculation at the cost of additional acquisition time.

Depending on the reaction process of interest for the particular control problem, visualizing and setting the gates that define the fitness regions can be done in one of two ways. First, as shown in Fig. 4, gates can be defined by radius and angle directly on the 3D-VMI itself. This is a good choice if

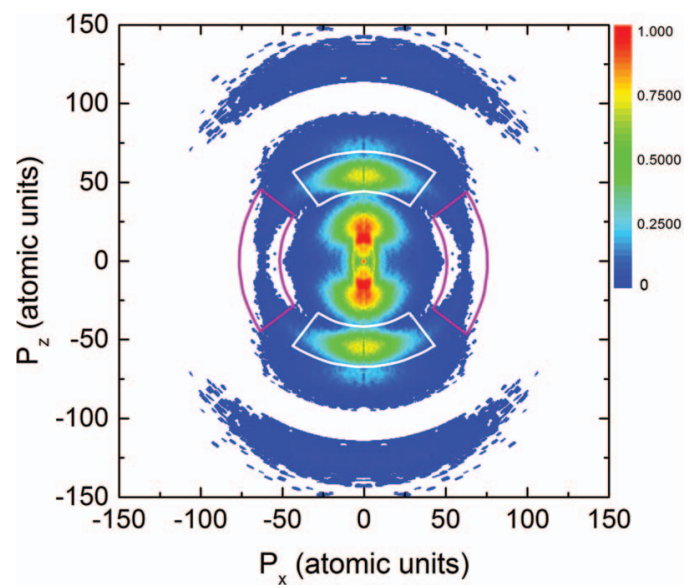

FIG. 4. $\mathrm{O}^{+}$photofragment $3 \mathrm{D}-\mathrm{VMI}$ data following ionization of $\mathrm{O}_{2}$ by a 45 fs, $\approx 1 \times 10^{14} \mathrm{~W} / \mathrm{cm}^{2}$ laser pulse with a center wavelength of $790 \mathrm{~nm}$. The outer ring, with radius between 100 and 150 atomic units, is dissociation of $\mathrm{O}_{2}^{2+} \rightarrow \mathrm{O}^{+}+\mathrm{O}^{+}$while the inner structures represent dissociation of $\mathrm{O}_{2}^{+}$to $\mathrm{O}^{+}+\mathrm{O}$ via 1,2 , and 3 additional photons. ${ }^{55-57}$ The laser polarization direction is vertical. The gate settings for this experiment, discussed in Sec. VI, are chosen to optimize the parallel ionization (yield in the white gates) vs. the perpendicular ionization (yield in the magenta gates) at KER of approximately $1.5 \mathrm{eV}$.

the angular distribution is a primary consideration. If a specific range of KER is of primary concern, it is sometimes easier to work with a plot that has already been converted to KER. In this case, we switch to a plot of yield vs. $\cos (\theta)$ and KER. In these plots we place the events with $\cos (\theta)= \pm 1$ (events parallel to the laser polarization) in the middle of the $y$-axis with $-1 \leq \cos (\theta) \leq 0$ events in the bottom part of the image and $0 \leq \cos (\theta) \leq 1$ events on the top of the image. This is shown in Fig. 5.

While the representations in Figs. 4 and 5 in principle contain the same information, we find that it is worth the extra effort to produce the different visualizations so that the
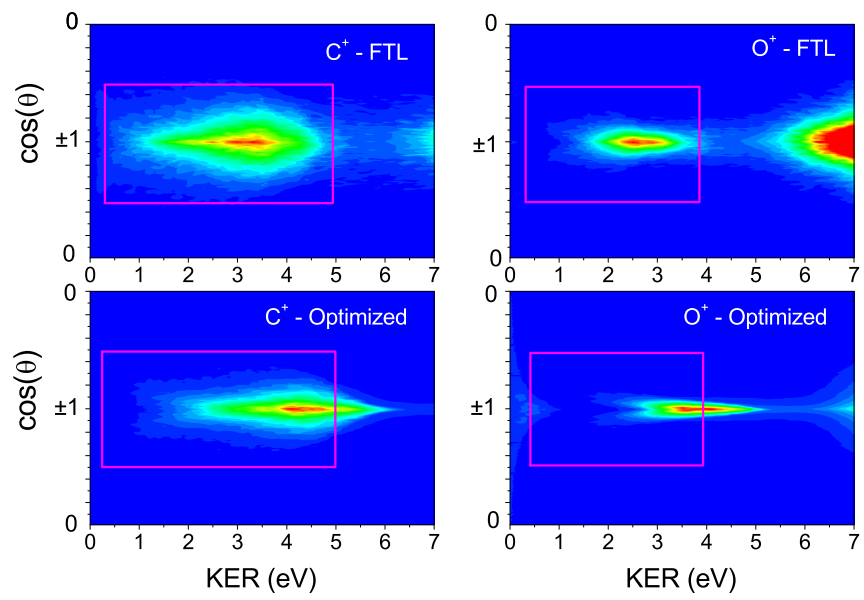

FIG. 5. Density plots as a function of both $\cos (\theta)$ and $\mathrm{KER}$ for $\mathrm{C}^{+}$(left) and $\mathrm{O}^{+}$(right) photofragments from $\mathrm{CO}$. Gates are defined by the regions inside the violet lines on each image. The top figures are obtained with FTL pulses and the bottom with an optimized pulse. Note that the optimized pulse has driven a significant amount of the $\mathrm{O}^{+}$signal out of the gate used in the fitness calculation. In this example, the fitness function was the ratio $\mathrm{C}^{+} / \mathrm{O}^{+}$, and thus reducing the measured $\mathrm{O}^{+}$yield in this manner enhanced the fitness value. 
experimenter can make the best choice of gate selection. This is especially true if KER is critical, as the fact that KER is proportional to momentum squared can easily lead to an asymmetric KER gate if great care is not taken. Once the yields in the particular areas are established using either set of gates, the experiment-specific fitness function is used to define the degree to which each trial pulse satisfies the goal of the experiment. Often, the fitness function takes the form of a ratio of two angle-resolved yields, such as $\mathrm{CH}_{2}^{+} / \mathrm{CH}^{+}$in the acetylene case. ${ }^{25}$ To protect against division by zero in the case of no signal, we add an offset to the denominator of these ratios that is about $10 \%$ of the size of the denominator signal when a FTL pulse is present.

In practice, we have found that selecting the gate settings that define the fitness function often requires some trial and error. Initially, we often begin by identifying a feature on an image obtained with a FTL laser pulse. In some cases (e.g., our acetylene work ${ }^{25}$ ), the identification of these features is guided by previous results in the literature. Depending on the desired control outcomes, gates that are set too tightly can provide results that are open to interpretation. An illustration of this is given in Fig. 5, in which we sought to enhance the ratio of $\mathrm{C}^{+}+\mathrm{O}$ to $\mathrm{C}+\mathrm{O}^{+}$from the dissociation of $\mathrm{CO}^{+}$. The ratio improved substantially, a result that cannot be duplicated with time-of-flight based feedback, but this was related to a shift in KER of the $\mathrm{O}^{+}$fragments rather than a change in the overall yield of $\mathrm{O}^{+}$fragments. As discussed in Sec. VI, this type of result may or may not be desirable.

\section{GENETIC ALGORITHM AND PULSE PARAMETERIZATIONS}

Using the experimental results as feedback, we employ the GA to search for a set of laser pulses that best achieve some defined target associated with the molecular process of interest. The search algorithm specifies a "genetic code" for each laser pulse, or "individual." The pulses may be parameterized in several ways as described below.

To begin the search, a "generation" of 30-50 individuals is created from random genetic sequences. The single-valued fitness of each individual in the generation is assessed according to its performance in achieving the specified target goal, as specified by the fitness function. Individual pulses are selected to "mate" based upon a tournament selection operator. Individuals with higher fitness have a greater chance of being selected for reproduction. In some cases the raw fitness is scaled, as suggested by Pearson et al. ${ }^{4}$ before the tournament selection process in order to increase the selection pressure and prevent stagnation of the search when similar fitness values are obtained for many different pulses. When the scaled fitness is used, the best pulse in the generation is twice as likely to be a parent than the average pulse.

Subsequent generations of pulses are constructed by mating the identified parents using either a two-point crossover operator or blend crossover operator. Most GAs use some form of the $n$-point crossover, in which a portion of the gene string from the parents is snipped and then spliced together to form the child. In two-point crossover, this process happens in two places. While two-point crossover exchanges parent gene values, the blend crossover operator allows for the creation of new gene values within the range between the parents' gene values. In this case, if one parent is defined by the gene string $A_{1}, A_{2}, \ldots, A_{n}$ and the other parent by $B_{1}, B_{2}, \ldots, B_{n}$, the value of the child's $C_{2}$ gene (for example) would be selected randomly from the range between $A_{2}$ and $B_{2}$. The main attribute of the blend operator is that it brings additional gene values into the search if there is concern that the initial population of genes might be too small to adequately sample the complete set of values.

In conjunction with the mating operators, a mutation operator introduces some probability for a random gene value to appear. The mutation rate is usually set at $1 \%$ per gene in these applications. Together, the mating and mutation operators define the genetic code of the offspring. We also use an elitism operator to add a genetic copy of the fittest individual into the next generation. The fitness of the individuals in the subsequent generation is then assessed using the same experimental technique, and the process is repeated until the GA converges on a solution. To check for experimental stability, we record the fitness for a FTL pulse between each generation. Most runs converge within 20-25 generations, although some find solutions more rapidly.

Two methods of gene parameterizations are used to describe the phase mask applied in the pulse shaping process. In the frequency parameterizations case, the genetic code is simply the phase delay at 16 points spread across the bandwidth of the pulse. These values become the 16 genes on the chromosome that identifies our individual pulses. The applied phase delay is linearly interpolated between each of these 16 points. In theory, defining more genes would result in finer control over the pulse. In practice, however, moderately restricting the number of genes ensures the algorithm begins to converge earlier and also prevents rapid phase oscillations which can stress the pulse shaping and amplification equipment. The linear interpolation also produces much the same result as the smoothing operator used by other groups, ${ }^{4}$ helping the subsequent pulse interpretation by limiting random fluctuation of gene values. If the spectral phase and amplitude are both shaped, the method is similar, with 8 (or 16) genes specifying the phase and a similar number specifying the amplitude.

The time-domain parameterization allows the shaped pulse to be built out of a series of $n$ Gaussian-shaped pulses, ${ }^{14}$ which can reduce the phase space of the search and is useful for identifying mechanisms that depend on the time interval between pulses. Implementation of the iterative Fouriertransform algorithm developed by Hacker and co-workers ${ }^{58}$ yields the intensity as a function of time while only physically controlling the spectral phase delay.

\section{SAMPLE APPLICATIONS}

Our recent work with small hydrocarbons ${ }^{25}$ illustrates two initial applications of 3D momentum-based feedback for adaptive control. Here we discuss two additional examples related to Figs. 4 and 5. Both examples will be discussed using a diabatic field-dressed (Floquet) representation, ${ }^{21,55,59,60}$ 


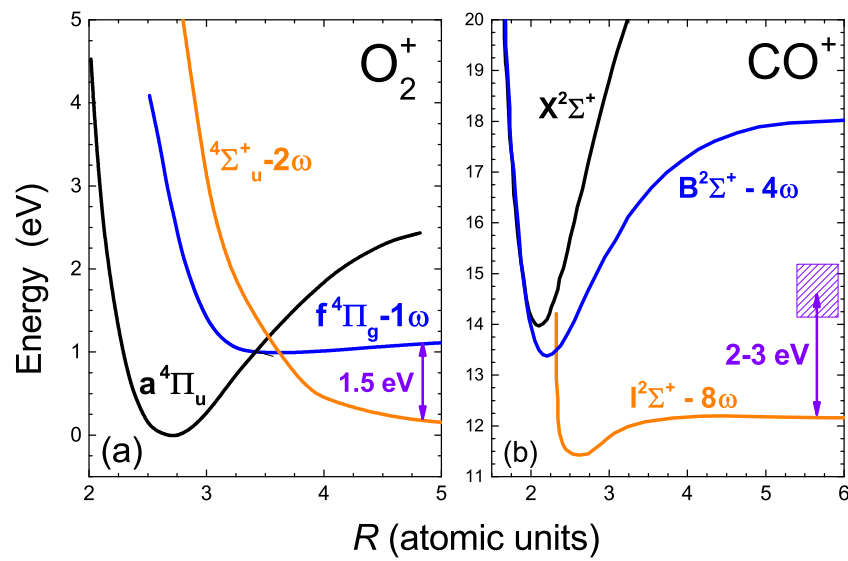

FIG. 6. Relevant potential energy curves for the dissociation pathways of (a) $\mathrm{O}_{2}^{+}$and (b) $\mathrm{CO}^{+}$as discussed in the text. Details of the potential energy curves may be found in Sayler et al. ${ }^{55}$ and Jochim et al., ${ }^{21}$ respectively, and references therein.

under the assumption that the process involves ionization followed by a dissociation.

Strong-field ionization of $\mathrm{O}_{2}$ leads to a number of dissociation pathways ${ }^{55-57,60}$ as shown by the data in Fig. 4. In one of these pathways, shown in Fig. 6(a), following ionization of $\mathrm{O}_{2}$ to the $a^{4} \Pi_{u}$ state of $\mathrm{O}_{2}^{+}$, the molecular ion dissociates via the $\left|a^{4} \Pi_{u}\right\rangle \rightarrow\left|f^{4} \Pi_{g}-1 \omega\right\rangle \rightarrow\left|{ }^{4} \Sigma_{u}^{+}-2 \omega\right\rangle$ pathway $^{55,56}$ to $\mathrm{O}^{+}+\mathrm{O}$ leading to a KER peak of around $1.5 \mathrm{eV}$. This "double bond-softening" pathway has a complex angular structure ${ }^{55}$ that includes both parallel (with the lasermolecule interaction depending on the laser field strength parallel to the internuclear axis since there is no change, $\Delta \Lambda=0$, in the angular momentum quantum number) and perpendicular $(\Delta \Lambda= \pm 1$, depending on the laser field strength perpendicular to the internuclear axis) transitions. While this is most likely the dominant pathway at this KER value, the $\left|a^{4} \Pi_{u}\right\rangle$ $\rightarrow\left|f^{4} \Pi_{g}-1 \omega\right\rangle$ bond-softening pathway should contribute a broad KER tail in this region. ${ }^{55,56,60}$ Other pathways, such as $\left|X^{2} \Pi_{g}\right\rangle \rightarrow\left|A^{2} \Pi_{u}-5 \omega\right\rangle$ (not shown), should contribute for high intensity pulses. The main $\left|a^{4} \Pi_{u}\right\rangle \rightarrow\left|f^{4} \Pi_{g}-1 \omega\right\rangle$ $\rightarrow\left|{ }^{4} \Sigma_{u}^{+}-2 \omega\right\rangle$ pathway is expected to have a $\cos ^{2}(\theta) \sin ^{2}(\theta)$ angular distribution, ${ }^{55,56,60}$ while the other pathways mentioned above should have angular distributions characterized by $\cos ^{2}(\theta)$ to $\cos ^{10}(\theta)$ depending on the number of photons involved.

Producing a feedback signal that could be used to discriminate these channels (all with KER of around $1.5 \mathrm{eV}$ ) based on standard time-of-flight techniques would be difficult. Using our method and the feedback gates shown in Fig. 4 , we can control the angular distribution of the fragments and thus select between pathways. The angle-resolved yields used in the fitness function are derived from the regions illustrated by the shapes shown in Fig. 4. In this example, we attempted to select between perpendicular and parallel transitions. The dissociation perpendicular to the laser polarization was suppressed by approximately $20 \%$ relative to the parallel dissociations. The mixed $\cos ^{2}(\theta) \sin ^{2}(\theta)$ character of the angular distribution of the main double bond-softening channel around KER of $1.5 \mathrm{eV}$ most likely accounts for our inability to produce a higher perpendicular to parallel contrast.

By combining our knowledge of the pathways from coincidence experiments ${ }^{55,56,60}$ with the image-based feedback approach described in this article, we can construct welldefined fitness functions that can target very specific objectives. For example, selection between the bond-softening and double bond-softening channels described above could be accomplished using the angular information to enhance or suppress yield around $\mathrm{KER}=1.5 \mathrm{eV}$ and $\theta=30^{\circ}$ relative to the same KER at $\theta=0^{\circ}$. In addition, VMI data can reveal vibrational structure, as demonstrated by this spectrometer with $\mathrm{O}_{2}$ targets and shorter laser pulses. ${ }^{56,57}$ This capability opens the possibility of vibrational level control using ion detection.

We have also applied the technique in the dissociative ionization of $\mathrm{CO},{ }^{21}$ illustrated in Fig. 5. In the experiment associated with Fig. 5, we influenced the ratio of $\mathrm{C}^{+}+\mathrm{O}$ to $\mathrm{C}+$ $\mathrm{O}^{+}$from the dissociation of $\mathrm{CO}^{+}$. The gates are set to include KER values associated with $\mathrm{CO}^{+}$and exclude higher KER dissociations from $\mathrm{CO}^{2}+$. In our original attempts to control this process ${ }^{21}$ with $2 \mathrm{D}$ image-based feedback, the background from these high KER photofragments obscured the low KER areas of interest. With 3D-VMI feedback, the control objective remained free of this background. Interestingly, however, the optimized pulse shifts $\mathrm{O}^{+}$population to higher KER, outside the gate, and away from the KER peak (identified as $\beta$ in Fig. 5 of Jochim et al. ${ }^{21}$ ) around $2-3 \mathrm{eV}$. The " $\beta$ " peak is suspected to arise from dissociation of $\mathrm{CO}^{+}$via the $\left|X^{2} \Sigma^{+}\right\rangle$ $\rightarrow\left|B^{2} \Sigma^{+}-4 \omega\right\rangle \rightarrow\left|I^{2} \Sigma^{+}-8 \omega\right\rangle$ pathway as shown in Fig. 6(b), with the width in KER accounted for by the substantial overlap of the $\left|X^{2} \Sigma^{+}\right\rangle$and $\left|B^{2} \Sigma^{+}-4 \omega\right\rangle$ states. The narrow angular distribution of the $\mathrm{O}^{+}$fragments just outside of the gate is not consistent with some of the lower-lying dissociative $\mathrm{CO}^{2+}$ states, ${ }^{61}$ suggesting that the optimized pulse is populating an excited electronic state of $\mathrm{CO}^{+}$while avoiding population of some $\mathrm{CO}^{2}+$ states, as indicated by the disappearance of $\mathrm{C}^{+}$and $\mathrm{O}^{+}$fragments with KER of $6-9 \mathrm{eV}$ in our data. While not shown in Fig. 5, the optimized pulse produces a substantial number of $\mathrm{C}^{+}$and $\mathrm{O}^{+}$fragments starting with KER values of around $9 \mathrm{eV}$ and extending to approximately $18 \mathrm{eV}$. Our analysis is hampered by a lack of available potential energy curves for $\mathrm{CO}^{+}$that dissociate to $\mathrm{C}+\mathrm{O}^{+}$ at the separated atom limit. Nevertheless, this example again demonstrates that the combination of KER and angular feedback capabilities in this experimental technique, when coupled with knowledge of molecular potential energy curves, can readily target strong-field dissociation pathways for control.

\section{SUMMARY AND FUTURE DIRECTIONS}

Feedback from 3D-VMI data can enhance adaptive femtosecond control by identifying well-defined control objectives and by providing a wealth of information that can help identify the mechanisms underlying the control. Obtaining this feedback requires the rapid inversion of the raw 2D-VMI data as well as robust computer control linking the different parts of the control loop. Careful implementation of the onion-peeling algorithm ${ }^{42}$ to maximize computational speed, 
coupled with the development of associated computer control, has achieved these goals and led to an initial set of successful experiments reported here and in Ref. 25.

The coupling of rapid 3D momentum feedback and ultrafast pulse shaping opens a number of new experimental avenues for coherent control. While our initial work focused on photodissociation, in principle, this method may be applied to any laser-driven system that can be measured using an imaging technique. Some interesting possibilities include rescattering electrons, ${ }^{62,63}$ nanoplasmas, ${ }^{64}$ nanodroplets, ${ }^{65}$ laserinduced molecular motions ${ }^{66}$ and excitations, ${ }^{67}$ and finally the possibility of one day using laser-induced electron diffraction of molecules ${ }^{62,68}$ or photoelectron tomography ${ }^{69}$ as control targets. Given the general applicability of 3D-VMI feedback and the context-rich information that can be gleaned from these images, there are undoubtedly additional directions that might be explored, especially in more complex systems.

We have begun work to add pump-probe capabilities to these experiments by shaping the pulse using a spatial light modulator $^{70}$ after the multi-pass amplifier, rather than between the laser oscillator and amplifier as in the current configuration. Pump-probe experiments, with potentially either the pump or the probe pulse shaped using adaptive control with image-based feedback, should in principle allow separation of the ionization and dissociation steps, further clarifying the control mechanism. For example, in a polyatomic molecule such as ethylene, the initial pulse might be used to stimulate an electronic excitation and the shaped secondary pulse might manipulate the nuclear wavepacket through an available conical intersection, ${ }^{71}$ thus exerting rapid and efficient control of the electronic to nuclear energy conversion. Three-dimensional image-based feedback should make identifying the exit pathways ${ }^{72,73}$ from the conical intersection plausible. Continuing advances in VMI and related techniques ${ }^{74,75}$ might also suggest future possibilities for more refined feedback for adaptive control.

\section{ACKNOWLEDGMENTS}

Augustana personnel, travel, and equipment were funded by the National Science Foundation under Grant Nos. PHY0969687 and EPS-0903804. T.G.B. and E.W. received additional support from the South Dakota Space Grant. JRML operations and personnel (including partial sabbatical leave for E.W.) were supported by the Chemical Sciences, Geosciences, and Biosciences Division, Office of Basic Energy Science, Office of Science, US Department of Energy. B.B., I.Z., and M.F.K. received partial support from the DFG via Grant No. Kl-1439/3 and the Cluster of Excellence "Munich Centre for Advanced Photonics" (MAP). We thank the referee for calling our attention to the maximum entropy method. ${ }^{54}$

\section{APPENDIX: COMPUTER CONTROL}

Experiments such as those described in Sec. VI generally must be automated in order to be practical, and thus require a high degree of coordination between several different hardware systems such as the AOPDF, detector power supply, and the camera readout electronics. In addition, various software processes, such as the GA and the image inversion algorithm, must be incorporated into the loop. In order to manage these operations, we have constructed a control program, based in the LabVIEW ${ }^{51}$ programming environment, that links the different systems. The various linkages in our setup are illustrated in Fig. 7.

The main program controls a number of sub-program elements. The camera control was built from the vendor-supplied LabVIEW code, as was the link to the digital delay generator that triggers the power to the microchannel plates. The proprietary AOPDF control code is initiated by the transfer of a text file that contains the path to the location of the pulse genome, and the pulse parameters are contained in one or more additional text files. The GA produces the values for these files which the master program then writes to the appropriate directory. In our initial work, the GA code was adapted from a freely available $\mathrm{C}++$ GA library ${ }^{76}$ and ran as a Windows console application that also communicated via text file. In the current variation, both the GA and the inversion routine are written in MATLAB and called from the LabVIEW code via the "script node" function, which has proved easier to edit. The main program also handles the image background subtraction and data storage, and displays the current run parameters.

Front panel inputs and controls include the delay generator settings that determine the ions to be examined, image acquisition time and number of images to be collected, option to start from a suspended run, and necessary parameters for the GA: the definition of fitness function, the number of generations, number of individuals per generation, selection of the gene parameterization scheme, mutation rate, an elitism toggle, and mating method. A typical screenshot from the program front panel is shown in Fig. 8. The LabVIEW-

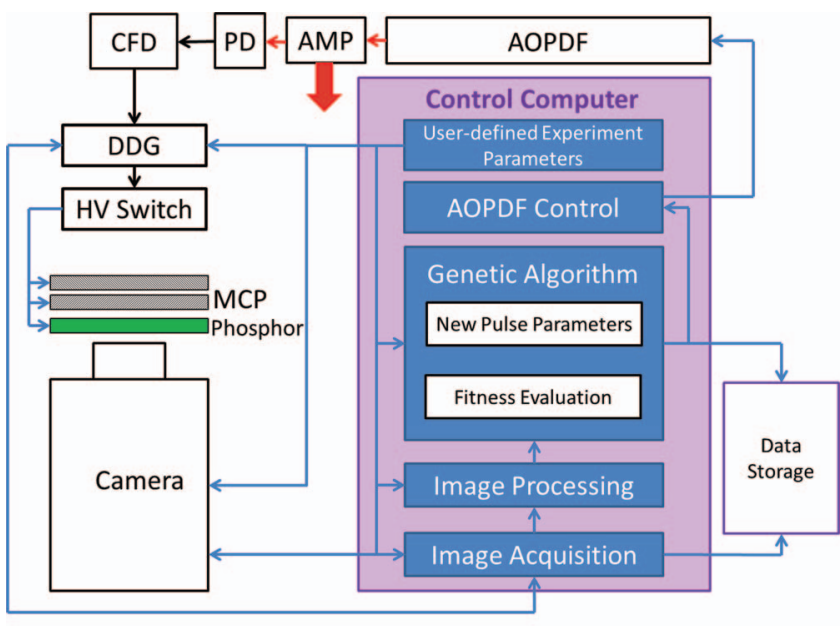

FIG. 7. Block diagram of the relationship between program elements. The shaped laser pulse from the AOPDF is amplified (AMP) and sent to the experiment. A portion of the laser pulse is measured with a photodiode (PD), processed by a constant fraction discriminator (CFD) and used to trigger the digital delay generator (DDG). The computer control coordinates the role of three instruments: The AOPDF, the camera, and the DDG. Event-driven program structure ensures the sequencing of the steps in the experiment. The image processing block includes the subtraction of background to remove any ambient light on the detector and the image inversion described in Sec. III. 


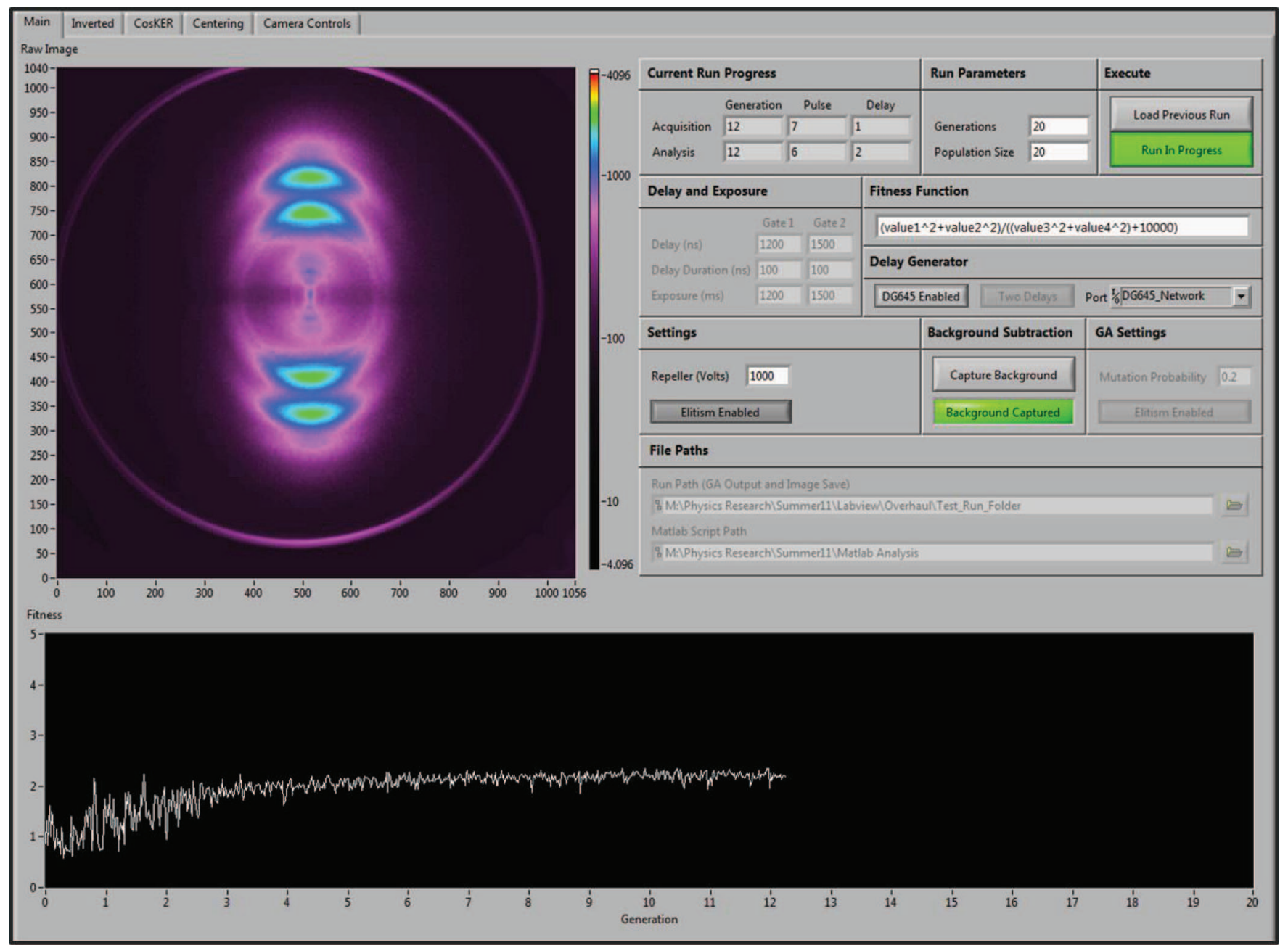

FIG. 8. Screen shot of the program front panel. The user interface is detailed and intuitive, allowing relatively simple initiation and monitoring of experiments. The main image in the upper left shows the raw (non-inverted) image most recently captured by the camera. Run parameters and equipment settings are displayed at the top right, while the bottom graph shows the evolution of the fitness function over time. Other display tabs show the inverted image and yield vs. $\cos (\theta)$ and KER. Either of these panels can be used to define and visualize the gates to be used in the fitness calculation. A fourth tab shows a full $1054 \times 1040$ pixel image for centering and the last tab contains the camera controls and temperature display.

based control code has an event-driven structure to simplify the execution of sequential steps in the process. This also allows the acquisition of an image to begin while the previous image is still being processed.

In these sorts of experiments, robust and flexible computer control is critical for success as both experimental parameters and the equipment change often in our circumstances. In experimental situations in which laser time is a valuable commodity, both the user interface and the ability to quickly understand how to modify the code are important. Given the significant (often predominant) role of undergraduates in this work, nearly all of the code is deliberately built from commercial software packages ${ }^{50,51}$ that are common in undergraduate educational settings, including Augustana College.

While the LabView application is quite specific to our apparatus due to the requirements of interfacing with individual pieces of equipment, the rapid inversion routine is fairly general and contained in several MATLAB m-files. Interested research groups may contact the corresponding author to obtain the code.
${ }^{1}$ R. Chakrabarti and H. Rabitz, Int. Rev. Phys. Chem. 26, 671 (2007).

${ }^{2}$ C. Brif, R. Chakrabarti, and H. Rabitz, New J. Phys. 12, 075008 (2010).

${ }^{3}$ A. Monmayrant, S. Weber, and B. Chatal, J. Phys. B: At., Mol. Opt. Phys. 43, 103001 (2010).

${ }^{4}$ B. J. Pearson, J. L. White, T. C. Weinacht, and P. H. Bucksbaum, Phys. Rev. A 63, 063412 (2001).

${ }^{5}$ J. Herek, W. Wohlleben, R. Cogdell, D. Zeidler, and M. Motzkus, Nature (London) 417, 533 (2002).

${ }^{6}$ R. Levis, G. Menkir, and H. Rabitz, Science 292, 709 (2001).

${ }^{7}$ J. Eland, Mol. Phys. 61, 725 (1987).

${ }^{8}$ M. J. Besnard-Ramage, P. Morin, T. Lebrun, I. Nenner, M. J. HubinFranskin, J. Delwiche, P. Lablanquie, and J. H. D. Eland, Rev. Sci. Instrum. 60, 2182 (1989).

${ }^{9}$ I. Ben-Itzhak, S. Ginther, and K. Carnes, Nucl. Instrum. Methods Phys. Res., Sect. B 66, 401 (1992).

${ }^{10}$ I. Ben-Itzhak, K. Carnes, and B. DePaola, Rev. Sci. Instrum. 63, 5780 (1992).

${ }^{11}$ R. Dörner, V. Mergal, L. Spielberger, J. Ullrich, R. Moshammer, and H. Schmidt-Böcking, Phys. Rep. 330, 95 (2000).

${ }^{12}$ J. Ullrich, R. Moshammer, A. Dorn, R. Dorner, L. P. H. Schmidt, and H. Schmidt-Bocking, Rep. Prog. Phys. 66, 1463 (2003).

${ }^{13}$ E. Wells, J. McKenna, A. Sayler, B. Jochim, N. Gregerson, R. Averin, M. Zohrabi, K. Carnes, and I. Ben-Itzhak, J. Phys. B: At., Mol. Opt. Phys. 43, 015101 (2010).

${ }^{14}$ E. Wells, K. J. Betsch, C. W. S. Conover, M. J. DeWitt, D. Pinkham, and R. R. Jones, Phys. Rev. A 72, 063406 (2005). 
${ }^{15}$ D. Chandler and P. Houston, J. Chem. Phys. 87, 1445 (1987).

${ }^{16}$ A. Eppink and D. Parker, Rev. Sci. Instrum. 68, 3477 (1997).

${ }^{17} \mathrm{~B}$. Whitaker, Imaging in Molecular Dynamics: Technology and Applications (A User's Guide) (Cambridge University Press, 2003).

${ }^{18}$ D. Geißler, T. Rozgonyi, J. González-Vázquez, L. González, P. Marquetand, and T. C. Weinacht, Phys. Rev. A 84, 053422 (2011).

${ }^{19}$ D. Irimia and M. H. M. Janssen, J. Chem. Phys. 132, 234302 (2010).

${ }^{20}$ A. Natan, U. Lev, V. S. Prabhudesai, B. D. Bruner, D. Strasser, D. Schwalm, I. Ben-Itzhak, O. Heber, D. Zajfman, and Y. Silberberg, Phys. Rev. A 86, 043418 (2012).

${ }^{21}$ B. Jochim, R. Averin, N. Gregerson, J. McKenna, S. De, D. Ray, M. Zohrabi, B. Bergues, K. D. Carnes, M. F. Kling, I. Ben-Itzhak, and E. Wells, Phys. Rev. A 83, 043417 (2011).

${ }^{22}$ G.-Y. Chen, Z. W. Wang, and W. T. Hill, Phys. Rev. A 79, 011401 (2009).

${ }^{23}$ M. Krug, T. Bayer, M. Wollenhaupt, C. Sarpe-Turdoran, T. Baumert, S. Ivanov, and N. Vitanov, New J. Phys. 11, 105051 (2009).

${ }^{24}$ O. Ghafur, A. Rouzee, A. Gijsbertsen, W. K. Siu, S. Stolte, and M. J. Vrakking, Nat. Phys. 5, 289 (2009).

${ }^{25}$ E. Wells, C. E. Rallis, M. Zohrabi, R. Siemering, B. Jochim, P. R. Andrews, U. Ablikim, B. Gaire, S. De, K. D. Carnes, B. Bergues, R. de Vivie-Riedle, M. F. Kling, and I. Ben-Itzhak, Nat. Commun. 4, 2895 (2013).

${ }^{26}$ J. Roslund and H. Rabitz, Phys. Rev. A 80, 013408 (2009).

${ }^{27}$ F. Verluise, V. Laude, Z. Cheng, C. Spielmann, and P. Tournois, Opt. Lett. 25, 575 (2000).

${ }^{28}$ R. Trebino, Frequency-Resolved Optical Gating (Kluwer Academic Publishers, 2000).

${ }^{29}$ S. De, I. Znakovskaya, D. Ray, F. Anis, N. G. Johnson, I. A. Bocharova, M. Magrakvelidze, B. D. Esry, C. L. Cocke, I. V. Litvinyuk, and M. F. Kling, Phys. Rev. Lett. 103, 153002 (2009).

${ }^{30}$ D. Ray, F. He, S. De, W. Cao, H. Mashiko, P. Ranitovic, K. P. Singh, I. Znakovskaya, U. Thumm, G. G. Paulus, M. F. Kling, I. V. Litvinyuk, and C. L. Cocke, Phys. Rev. Lett. 103, 223201 (2009).

${ }^{31}$ N. G. Kling, D. Paul, A. Gura, G. Laurent, S. De, H. Li, Z. Wang, B. Ahn, C. H. Kim, T. K. Kim, I. Litvinyuk, C. L. Cocke, I. Ben-Itzhak, D. Kim, and M. F. Kling, J. Instrum. 9, P05005 (2014).

${ }^{32}$ O. Ghafur, W. Siu, P. Johnsson, M. Kling, M. Drescher, and M. Vrakking, Rev. Sci. Instrum. 80, 033110 (2009).

${ }^{33}$ N. Abel, Oeuvres Completes (Johnson Reprint Corp., New York, 1988).

${ }^{34}$ C. Dasch, Appl. Opt. 31, 1146 (1992).

${ }^{35} \mathrm{R}$. Bracewell, The Fourier Transform and its Applications (McGraw-Hill, New York, 1978).

${ }^{36}$ C. R. Gebhardt, T. P. Rakitzis, P. C. Samartzis, V. Ladopoulos, and T. N. Kitsopoulos, Rev. Sci. Instrum. 72, 3848 (2001).

${ }^{37}$ J. J. Lin, J. Zhou, W. Shiu, and K. Liu, Rev. Sci. Instrum. 74, 2495 (2003).

${ }^{38}$ D. Townsend, M. P. Minitti, and A. G. Suits, Rev. Sci. Instrum. 74, 2530 (2003).

${ }^{39}$ W. Castleman, Digital Image Processing (Prentice-Hall, London, 1979).

${ }^{40}$ S. Candel, Comput. Phys. Commun. 23, 343 (1981).

${ }^{41}$ L. Smith and D. Keefer, J. Quant. Spectrosc. Radiat. Transfer 39, 367 (1988).

${ }^{42}$ C. Bordas, F. Paulig, H. Helm, and D. L. Huestis, Rev. Sci. Instrum. 67, 2257 (1996).

${ }^{43}$ J. Winterhalter, D. Maier, J. Honerkamp, V. Schyja, and H. Helm, J. Chem. Phys. 110, 11187 (1999).

${ }^{44}$ K. Zhao, T. Colvin, W. T. Hill III, and G. Zhang, Rev. Sci. Instrum. 73, 3044 (2002).

${ }^{45}$ M. J. J. Vrakking, Rev. Sci. Instrum. 72, 4084 (2001).

${ }^{46}$ V. Dribinski, A. Ossadtchi, V. A. Mandelshtam, and H. Reisler, Rev. Sci. Instrum. 73, 2634 (2002).

${ }^{47}$ G. A. Garcia, L. Nahon, and I. Powis, Rev. Sci. Instrum. 75, 4989 (2004).

${ }^{48}$ F. Renth, J. Riedel, and F. Temps, Rev. Sci. Instrum. 77, 033103 (2006).

${ }^{49}$ G. Roberts, J. Nixon, J. Lecointre, E. Wrede, and J. Verlet, Rev. Sci. Instrum. 80, 053104 (2009).
${ }^{50}$ M. Inc., "Matlab," see http://www.mathworks.com/products/matlab/, 2009.

${ }^{51}$ N. I. Corp., "Labview," see http://www.ni.com/labview/, 2011.

${ }^{52}$ M. Apostolopoulos, M. Taroudakis, and D. Papazoglou, Opt. Commun. 296, 25 (2013).

${ }^{53}$ T. Gerber, Y. Liu, G. Knopp, P. Hemberger, A. Bodi, P. Radi, and Y. Sych, Rev. Sci. Instrum. 84, 033101 (2013).

${ }^{54}$ B. Dick, Phys. Chem. Chem. Phys. 16, 570 (2014).

${ }^{55}$ A. M. Sayler, P. Q. Wang, K. D. Carnes, B. D. Esry, and I. Ben-Itzhak, Phys. Rev. A 75, 063420 (2007).

${ }^{56}$ M. Zohrabi, J. McKenna, B. Gaire, N. G. Johnson, K. D. Carnes, S. De, I. A. Bocharova, M. Magrakvelidze, D. Ray, I. V. Litvinyuk, C. L. Cocke, and I. Ben-Itzhak, Phys. Rev. A 83, 053405 (2011).

${ }^{57}$ S. De, M. Magrakvelidze, I. A. Bocharova, D. Ray, W. Cao, I. Znakovskaya, H. Li, Z. Wang, G. Laurent, U. Thumm, M. F. Kling, I. V. Litvinyuk, I. Ben-Itzhak, and C. L. Cocke, Phys. Rev. A 84, 043410 (2011).

${ }^{58}$ M. Hacker, G. Strobrawa, and T. Feurer, Opt. Express 9, 191 (2001).

${ }^{59}$ G. Floquet, Ann. Ec. Norm. 13, 47 (1883).

${ }^{60}$ A. Hishikawa, S. Liu, A. Iwasaki, and K. Yamanouchi, J. Chem. Phys. 114, 9856 (2001).

${ }^{61}$ J. McKenna, A. M. Sayler, F. Anis, N. G. Johnson, B. Gaire, U. Lev, M. A. Zohrabi, K. D. Carnes, B. D. Esry, and I. Ben-Itzhak, Phys. Rev. A 81, 061401 (2010).

${ }^{62}$ Y. Huismans, A. Rouze, A. Gijsbertsen, J. H. Jungmann, A. S. Smolkowska, P. S. W. M. Logman, F. Lpine, C. Cauchy, S. Zamith, T. Marchenko, J. M. Bakker, G. Berden, B. Redlich, A. F. G. van der Meer, H. G. Muller, W. Vermin, K. J. Schafer, M. Spanner, M. Y. Ivanov, O. Smirnova, D. Bauer, S. V. Popruzhenko, and M. J. J. Vrakking, Science 331, 61 (2011), see http://www.sciencemag.org/content/331/6013/61. full.pdf.

${ }^{63}$ D. D. Hickstein, P. Ranitovic, S. Witte, X.-M. Tong, Y. Huismans, P. Arpin, X. Zhou, K. E. Keister, C. W. Hogle, B. Zhang, C. Ding, P. Johnsson, N. Toshima, M. J. J. Vrakking, M. M. Murnane, and H. C. Kapteyn, Phys. Rev. Lett. 109, 073004 (2012).

${ }^{64}$ D. D. Hickstein, F. Dollar, J. A. Gaffney, M. E. Foord, G. M. Petrov, B. B. Palm, K. E. Keister, J. L. Ellis, C. Ding, S. B. Libby, J. L. Jimenez, H. C. Kapteyn, M. M. Murnane, and W. Xiong, Phys. Rev. Lett. 112, 115004 (2014).

${ }^{65}$ D. Pentlehner, J. H. Nielsen, A. Slenczka, K. Mølmer, and H. Stapelfeldt, Phys. Rev. Lett. 110, 093002 (2013).

${ }^{66}$ J. L. Hansen, J. H. Nielsen, C. B. Madsen, A. T. Lindhardt, M. P. Johansson, T. Skrydstrup, L. B. Madsen, and H. Stapelfeldt, J. Chem. Phys. 136, 204310 (2012).

${ }^{67}$ J. R. R. Verlet, D. A. Horke, and A. S. Chatterley, Phys. Chem. Chem. Phys. 16, 15043 (2014).

${ }^{68}$ C. Blaga, J. Xu, A. DiChiara, E. Sistrunk, K. Zhang, P. Agostini, T. Miller, L. DiMauro, and C. Lin, Nature (London) 483, 194 (2012).

${ }^{69}$ J. Maurer, D. Dimitrovski, L. Christensen, L. B. Madsen, and H. Stapelfeldt, Phys. Rev. Lett. 109, 123001 (2012).

${ }^{70}$ A. M. Weiner, Rev. Sci. Instrum. 71, 1929 (2000).

${ }^{71}$ B. Levine and T. Martinez, Ann. Rev. Phys. Chem. 58, 613 (2007).

${ }^{72}$ H. Tao, T. K. Allison, T. W. Wright, A. M. Stooke, C. Khurmi, J. van Tilborg, Y. Liu, R. W. Falcone, A. Belkacem, and T. J. Martinez, J. Chem. Phys. 134, 244306 (2011).

${ }^{73}$ T. K. Allison, H. Tao, W. J. Glover, T. W. Wright, A. M. Stooke, C. Khurmi, J. van Tilborg, Y. Liu, R. W. Falcone, T. J. Martnez, and A. Belkacem, J. Chem. Phys. 136, 124317 (2012).

${ }^{74}$ A. Vredenborg, W. G. Roeterdink, and M. H. M. Janssen, Rev. Sci. Instrum. 79, 063108 (2008).

${ }^{75}$ A. T. Clark, J. P. Crooks, I. Sedgwick, R. Turchetta, J. W. L. Lee, J. J. John, E. S. Wilman, L. Hill, E. Halford, C. S. Slater, B. Winter, W. H. Yuen, S. H. Gardiner, M. L. Lipciuc, M. Brouard, A. Nomerotski, and C. Vallance, J. Phys. Chem. A 116, 10897 (2012), see http://pubs.acs.org/ doi/pdf/10.1021/jp309860t.

${ }^{76}$ M. Wall, Massachusetts Institute of Technology (1999), see http://lancet.mit.edu/ga/. 